\title{
JOURNAL.RU
}

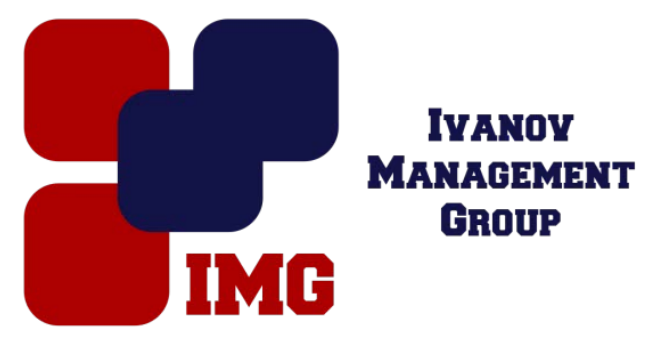

Омаркулов К.А, Акимбеков Е.Т. Казахский агротехнический университет имени С.Сейфуллина Астана, Республика Казахстан

doi: 10.18411/lj-31-03-2017-2-02

idsp 000001:lj-31-03-2017-2-02

\section{О модельной фотогравитационной задаче в нестационарном поле тяготения}

\section{Аннотация}

В данной статье рассматривается задача движение искусственного спутника в нестационарном поле тяготения и интегрирования уравнения Гамильтона-Якоби.

Ключевые слова: уравнения Гамильтона-Якоби, поля тяготения, постоянные интегрирования.

В настоящие время в литературе обсуждаются проекты интегрирования уравнения Гамильтона-Якоби. Как приложение рассмотрим задачу движение искусственного спутника в нестационарном поле тяготения.

В последние годы наблюдается интенсивное развитие идей Дирака об изменении гравитационной констаты $\mathrm{G}$ во времени как в плане ее дальнейшего теоретического обобщения, так и в отношении анализа возможных эффектов переменной гравитации в эволюции различных форм космической материи[1].Временнные вариации $G$ в принципе допускает экспериментальное определение[1-2]. В частности, можно попытаться учитывать вариации G в движении искусственного спутника земли. Как известно, на движение искусственных спутников земли оказывает влияние целый ряд возмущающих факторов. Важнейшим из них является неферичность земли. Учет этого фактора проводится на основе решения обобщенной задачи двух неподвижных центров, учитывающий сжатие земли[2]. В связи с тем,что гравитационная проблема Земля- спутник эквивалента с точностью до первых двух зональных гармоник геоптенциала обощенной задаче двух неподвижых центров и представяется интересным приводимое ниже промежуточное движение для нестационарной схемы этой задачи с переменнойгравитационной константы; 


$$
G(t)=\frac{G_{0}}{\sqrt{1+\alpha t}}
$$

где $\alpha$ - постоянная, G0- гравитационная постоянная.

Выпишем силовую функцию Јобобщенной задачи двух неподвижных центров P1 и Р2 в барицентрической системе прямоугольных координат Охуzс осью аппликат вдоль линии Р1, P2 [2].

$$
U=\frac{G(t) m}{2} \frac{\{1+\sigma i}{r_{1}}+\frac{1-\sigma i\}}{r_{2}}+\varepsilon \cdot r^{2}
$$

причем $r_{1}^{2}=x^{2}+y^{2}+[z-c(\sigma+i)]^{2}, r_{2}^{2}=x^{2}+y^{2}+[z-c(\sigma+i)]^{2}, i^{2}=-1$

где m, би с - постоянные.

Не останавливаясь на выкладках, приведем общий-интеграл нашей задачи, получающейся согласно методу Гамильтона-Якоби [2].

$$
\begin{gathered}
c^{2} \int \frac{\lambda^{2} d \lambda}{\sqrt{L(\lambda)}}+c^{2} \int \frac{\mu^{2} d \mu}{\sqrt{M(\mu)}}=\int \frac{\sqrt{G_{0}} d t}{\sqrt{1+\alpha t}}+\beta_{1} \\
\int \frac{d \lambda}{\sqrt{L(\lambda)}}-c^{2} \int \frac{d \mu}{\sqrt{M(\mu)}}=\beta_{2} \\
\alpha_{3} \int \frac{d \lambda}{\left(1+\lambda^{2}\right) \sqrt{L(\lambda)}}-\alpha_{3} \int \frac{d \mu}{\left(1+\mu^{2}\right) \sqrt{M(\mu)}}+\omega=\beta_{31} \\
P_{\lambda}=\frac{G(t) \cdot L(\lambda)}{1+\lambda^{2}} P_{\mu}=\frac{G(t) \cdot M(\mu)}{1-\mu^{2}} P_{\omega}=\alpha_{3} \cdot \sqrt{G(t)}
\end{gathered}
$$

где $\mathrm{L}(\lambda)$ иМ $(\mu)$ определяется по формулам

$\mathrm{L}(\lambda)=2(1+\lambda 2)(\mathrm{hc} 2 \lambda 2+\mathrm{mc} \lambda+\alpha 2)+\alpha 32$ иM $(\mu)=2(1-\mu 2)($ hc $2 \mu 2-$ mc $\mu-\alpha 2)-$ $\alpha 32$

в которых $\mathrm{h}, \alpha 2, \beta 1, \beta 2, \beta 3$ с- постоянные интегрирования.

Формулы (3) и (4) дают полное решение рассматриваемой задачи о движении материальной точки в поле тяготения двух неподвижных центров переменной гравитации.

Таким образом, обобщенная задача двух неподвижных центров с переменной во времени постоянной тяготения $\mathrm{G}$ интегрируема при наличии добавочной силы, пропорциональной скорости пробного тела и относительной скорости изменения G. Найденное решение может быть использовано как промежуточное движение при анализе эффектов переменной гравитации и 
переменной массы в движении исследуемых тел в нецентральном поле тяготения.

\section{Литература}

1. Омаров Т.Б. Динамика гравитирующих систем Метогалактики., Алма-Ата, «Наука», 1975 г.

2. Демин В.Г. Движение искусственного спутника внецентральном поле тяготения. М., «Наука», 1968ж.

3. М.Д.Шинибаев, А.А.Беков, С.С.Дайырбеков, Д.И.Усипбекова Орбитальные движения пробного тела в центральном поле тяготения Хилла. // Вестник НАН РК №4, 2014 год. 